\title{
Nonlinear Control of Single-Phase Shunt Active Power filter Theoretical analysis of closed-loop performances
}

\author{
I. Lachkar ${ }^{1}$, F. Giri $^{2}$, A. Abouloifa ${ }^{1}$, J. M. Guerrero ${ }^{3}$, R. Grino ${ }^{3}$, F.Z. Chaoui ${ }^{1}$, H. Elfadil ${ }^{4}$ \\ ${ }^{1}$ LA2I, EMI, Rabat, Morocco \\ ${ }^{2}$ GREYC Lab, UMR CNRS, University of Caen, France, fouad.giri@unicaen.fr \\ ${ }^{3}$ Universitat Politècnica de Catalunya, Barcelona, Spain \\ ${ }^{4}$ LHESIR Lab, ENSA, Ibn Tofail University, Kenitra, Morocco
}

\begin{abstract}
The problem of controlling single-phase shunt active power filter is addressed in presence of nonlinear loads. The control objective is twofold: (i) compensation of harmonic and reactive currents absorbed by the nonlinear load; (ii) regulation of the inverter output capacitor voltage. A two-loop cascade control strategy is developed that includes an inner-loop designed, using the backstepping technique, to cope with the compensation issue and an outer-loop designed to regulate the capacitor voltage. The controller performances are formally analysed, using the averaging theory. The analysis results are illustrated by simulation.
\end{abstract}

\section{INTRODUCTION}

In presence of nonlinear loads, current harmonics are generated that cause the distortion of the voltage waveform at the point of common coupling (PCC), leading to voltage harmonics and disturbing other connected loads and electronic equipments. For a long time, the compensation of these harmonics has been sought using passive LC filters and capacitor banks. The latter were resorted to improve the AC loads power factor. This conventional solution presents several shortcomings. Then, efforts have been made, by engineers and researchers in power energy and power electronics, to develop alternative solutions to these power quality problems. In this respect, the concept of active power filter (APF) emerged a couple of decades ago (Gyugyi \& Strycula, 1976). Since then, many research programs have been conducted on active power filters and their practical applications. Nowadays, modern active power filters, compared to traditional passive filters, are superior in filtering performance, smaller in physical size and more flexible in application.

APFs can be divided into single phase and three phase active filters. Single phase APFs have attracted less attention than three phase APFs because they are limited to low power applications. However, implementing a low-power singlephase APF to each single-phase nonlinear load may be, in some situations, a better solution than installing a single medium or high power three-phase APF at the PCC.

In the last decade, the problem of controlling single-phase shunt APFs has been given a great deal of interest. In this respect, several control strategies have been proposed including simple fuzzy logic based control (Doğan \& Akkaya, 2009), linear control (Costa, 2007) and nonlinear control (Miret, 2004). One common shortcoming of most previous works is the lack of formal performance analysis backing the proposed control strategies. In this paper, a new control strategy is developed for single-phase shunt APFs in presence of nonlinear loads. Specifically, a nonlinear controller is proposed to ensure a satisfactory compensation of harmonic and reactive currents absorbed by the nonlinear load and a tight regulation of the inverter output capacitor voltage. The controller is a two-loop cascade structure involving an inner-loop designed using the backstepping technique, to cope with the compensation issue, and an outernonlinear PI control loop designed to regulate the output capacitor voltage. It is formally shown, using tools from the Lyapunov stability and averaging theories, that all control objectives are actually achieved. This theoretical result is confirmed by several numerical simulations.

The paper is organized as follows: the converter is modeled in Section 2; the control problem is formulated in Section 3 which also includes the controller design and analysis; the controller performances are illustrated by simulation in Section 4.

\section{ACTIVE POWER FILTER MODELING}

\subsection{APF Modelling}

Applying the Kirchhoff's laws to the single phase shunt APF one easily gets:

$$
\left\{\begin{array}{c}
L_{f} \frac{d i_{f}}{d t}=v_{s}-v_{A B} \\
C_{f} \frac{d v_{d c}}{d t}=i_{d c}
\end{array}\right.
$$

The inverter undergoes the equations:

$$
\left\{\begin{array}{c}
v_{A B}=\mu \cdot v_{d c} \\
i_{d c}=\mu . i_{f}
\end{array}\right.
$$

The switching function $\mu$ of the converter is defined by: 


$$
\mu=\left\{\begin{array}{ccc}
1 & \text { if }\left(s_{1}, s_{4}\right) \text { is } O N ;\left(s_{2}, s_{3}\right) \text { is } \text { OFF } \\
-1 \text { if }\left(s_{1}, s_{4}\right) \text { is OFF } ;\left(s_{2}, s_{3}\right) \text { is } O N
\end{array}\right.
$$

Combining (1) and (2), one obtains the instantaneous model of the filter:

$$
\left\{\begin{array}{c}
\frac{d i_{f}}{d t}=\frac{1}{L_{f}}\left(v_{s}-\mu v_{d c}\right) \\
\frac{d v_{d c}}{d t}=\frac{1}{C_{f}} \mu i_{f}
\end{array}\right.
$$

The model (4) is useful to build-up an accurate simulator for the converter. However, it cannot be based upon in the control design as it involves a binary control inputs, namely $\mu$. This difficulty is usually coped with resorting to average models and the averaging is performed over cutting intervals (Abouloifa et al., 2003). Accordingly, the mean value of $\mu$ turns out to be the system control input. The obtained average model is the following:

$$
\left\{\begin{array}{l}
\frac{d x_{1}}{d t}=\frac{1}{L_{f}}\left(v_{s}-u x_{2}\right) \\
\frac{d x_{2}}{d t}=\frac{1}{C_{f}} u x_{1}
\end{array}\right.
$$

where $x_{1}, x_{2}$ and $u$ denote the average values, over cutting periods, of the signals $i_{f}, v_{d c}$ and $\mu$. The system (5) is clearly nonlinear.

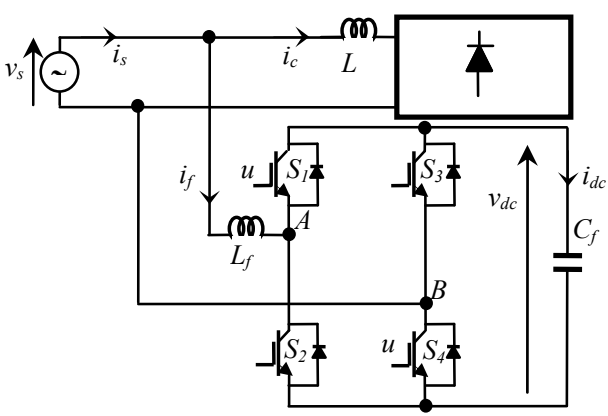

Fig.1. Shunt filter connected to the network-load system.

\section{CONTROLLER DESIGN AND ANALYSIS}

The controller synthesis is carried out in two major stages. First, a current inner loop is designed to cope with the power compensation issue. In the second step, an outer loop is builtup to achieve voltage regulation in the DC side of active power filter.

\subsection{Current inner loop design}

This aims at making the network current $i_{s}$ sinusoidal and in phase with the network voltage $v_{s}$. This means that $x_{1}$ should follow the reference $x_{1}^{*}$ defined by:

$x_{1}^{*}=\beta v_{s}-i_{c}$
For theoretical design considerations, it is more convenient to reformulate the above objective in term of the energy function $\xi(t)$ defined as follows:

$\xi(t)=\frac{L_{f}}{2} x_{1}^{2}+\frac{C_{f}}{2} x_{2}^{2}$

Using (5) and (7) it follows that that $\xi(t)$ undergoes the following equations:

$\dot{\xi}=v_{s} x_{1}$

$\ddot{\xi}=\dot{v}_{s} x_{1}+\frac{v_{s}}{L_{f}}\left(v_{s}-u x_{2}\right)$

It readily follows from equation (8) that:

$\xi=\int v_{s} x_{1} d \tau$

The control objective is now to make $\xi$ track as closely as possible the reference signal $\xi^{*}$, obtained substituting $x_{1}^{*}$ to $x_{1}$ in (10). Specifically:

$\xi^{*}=\int v_{s} x_{1}^{*} d t$

Doing so, the network current harmonics and reactive power will be cancelled. To this end, a regulator will now be designed in two steps, using the backstepping technique (Krstic et al., 1995).

Step 1. Introduce the tracking error on the energy $\xi$ :

$z_{1}=\xi-\xi^{*}$

Using (8), time-derivation of (11) yields:

$\dot{z}_{1}=v_{s}\left(x_{1}-x_{1}^{*}\right)$

In (12), the quantity $\left(v_{s} x_{1}\right)$ stands as a virtual control variable. Then, $z_{1}$ can be regulated to zero by letting $\left(v_{s} x_{1}\right)=\sigma_{1}$ where $\sigma_{1}$, called stabilizing function, is defined by:

$\sigma_{1}=-c_{1} z_{1}+v_{s} x_{1}^{*}$

where $c_{1}>0$ is a design parameter. Indeed, this choice would imply $\dot{z}_{1}=-c_{1} z_{1}$, ensuring the asymptotic stability of (12) with respect to the Lyapunov function:

$V_{1}=\frac{1}{2} z_{1}^{2}$

Indeed, time-derivation of $V_{1}$ would then imply:

$\dot{V}_{1}=-c_{1} z_{1}^{2}<0$

which is negative definite with respect to $z_{1}$. As $\left(v_{s} x_{1}\right)$ is not the actual control input, a new error variable, denoted $z_{2}$, between the virtual control and its desired value $\left(\sigma_{1}\right)$ is introduced: 


$$
z_{2}=\left(v_{s} x_{1}\right)-\sigma_{1}
$$

Then, equation (12) becomes, using (13) and (16):

$$
\dot{z}_{1}=-c_{1} z_{1}+z_{2}
$$

Also, the derivative of Lyapunov function (15) becomes:

$$
\dot{V}_{1}=-c_{1} z_{1}^{2}+z_{1} z_{2}
$$

Step 2. Achieving the control objective now amounts to enforcing the errors $\left(z_{1}, z_{2}\right)$ to vanish. To this end, one needs the dynamics of $z_{2}$. Deriving (16) and using (5a) yields:

$\dot{z}_{2}=\left(\dot{v}_{s}+c_{1} v_{s}\right) e+\frac{1}{L_{s}} v_{s}^{2}-\frac{1}{L_{s}} x_{2} v_{s} u-v_{s} \dot{x}_{1}^{*}$

with:

$$
e=x_{1}-x_{1}^{*}
$$

The actual control variable, namely $u$, has emerged for the first time in equation (19). An appropriate control law for generating $u$ must now be determined so that the $\left(z_{1}, z_{2}\right)$ system is made globally asymptotically stable. To this end, consider the Lyapunov function candidate:

$V_{2}=V_{1}+\frac{1}{2} z_{2}^{2}$

Using (18) and (20), the time-derivative of $V_{2}$ can be rewritten as:

$$
\dot{V}_{2}=-c_{1} z_{1}^{2}+z_{2}\left(z_{1}+\dot{z}_{2}\right)
$$

This shows that, for the $\left(z_{1}, z_{2}\right)$-system to be globally asymptotically stable, it is sufficient to choose the control $u$ so that $\dot{V}_{2}=-c_{1} z_{1}^{2}-c_{2} z_{2}^{2}$ which, due to (21), amounts to ensuring that:

$$
\dot{z}_{2}=-z_{1}-c_{2} z_{2}
$$

Comparing (22) and (23) yields the following backstepping control law:

$$
u=\frac{L_{f}}{x_{2}}\left[\frac{\left(\dot{v}_{s}+c_{1} v_{s}\right)}{v_{s}} e+\frac{1}{L_{f}} v_{s}-\dot{x}_{1}^{*}+\frac{z_{1}+c_{2} z_{2}}{v_{s}}\right]
$$

Using (11), (13) and (16), this control law can be expressed as follows:

$u=\frac{L_{f}}{x_{2}}\left[\left(\frac{\dot{v}_{s}}{v_{s}}+c_{1}+c_{2}\right) e+\frac{v_{s}}{L_{f}}-\dot{x}_{1}^{*}+\left(1+c_{1} c_{2}\right) \frac{\int v_{s} e d \tau}{v_{s}}\right]$ (24)

As the network voltage $v_{s}$ vanishes periodically, the control law (24) entails singularities. To overcome this issue the following modified version of (24) is proposed:

$$
u_{m}=\frac{L_{f}}{x_{2}}\left[\left(\frac{\dot{v}_{s}}{\hat{V}}+c_{1}+c_{2}\right) e+\frac{v_{s}}{L_{f}}-\dot{x}_{1}^{*}+\left(1+c_{1} c_{2}\right) \frac{\int \hat{V} \cdot e \cdot d \tau}{\hat{V}}\right]
$$

The above control law involves the derivative of the reference signal $x_{1}^{*}$. From (6) and (25), it follows that the ratio $\beta$ and its derivative must be available. The results thus established are summarized in the following proposition.

Proposition 1. Consider the system, next called inner closedloop, consisting of the system (5) and the control law (25). If the ratio $\beta$ and its first time-derivative are available, then the inner closed-loop system undergoes, in the $\left(e_{1}, e_{2}\right)$ coordinates, the following equation:

$\left(\begin{array}{c}\dot{e}_{1} \\ \dot{e}_{2}\end{array}\right)=\left(\begin{array}{cc}0 & 1 \\ -\left(1+c_{1} c_{2}-\omega_{s}^{2} \sin \omega_{s} t\right) & -\left(c_{1}+c_{2}+\omega_{s} \cos \omega_{s} t\right)\end{array}\right)\left(\begin{array}{l}e_{1} \\ e_{2}\end{array}\right)$

with $e_{1}=e$ and $e_{2}=\dot{e}$

Proof. Substituting $u_{m}$, generated by the control law (25), to $u$ in (the first equation of) (5) yields the following dynamics of the current error:

$\dot{e}=-\left(\omega_{s} \cos \left(\omega_{s} t\right)+c_{1}+c_{2}\right) e-\left(1+c_{1} c_{2}\right) \int e d \tau$

Deriving this wit respect to time gives:

$\ddot{e}+\left(c_{1}+c_{2}+\omega_{s} \cos \omega_{s} t\right) \dot{e}+\left(1+c_{1} c_{2}-\omega_{s}^{2} \sin \omega_{s} t\right) e=0$

which also takes the state space representation (28)

\subsection{Voltage outer loop design}

The aim of the outer loop is to generate a tuning law for the ratio $\beta$ in a way that makes the output voltage $x_{2}$ regulated to a given reference value $x_{2}^{*}$. To this end, the relation between $\beta$ and $x_{2}$ must be made clear. This is carried out in the following proposition. For simplicity, it is supposed that the inner loop is much faster than the outer loop, a condition that will be accounted for when tuning the parameters of the inner and outer loop.

Proposition 2. Consider the power active filter described by (7) in closed-loop with the inner control law defined by (27). Under the same assumptions as in Proposition 1, one has:

1) The output voltage $x_{2}$ varies in response to the tuning ratio $\beta$ according to the following equation:

$$
\begin{aligned}
\dot{x}_{2}= & -\frac{L_{f}}{2 C_{f} x_{2}} \hat{V}^{2}\left(1-\cos \left(2 \omega_{s} t\right)\right) \beta^{2} \\
& +\frac{1}{C_{f} x_{2}}\left[\hat{V}^{2}\left(1-\cos \left(2 \omega_{s} t\right)\right)+2 L_{f} v_{s} i_{c}+L_{f} \Delta \hat{V} \sin \omega_{s} t\right] \beta \\
& -\frac{L_{f}}{C_{f} x_{2}}\left(i_{c}^{2}+\frac{1}{L_{f}} v_{s} i_{c}+\Delta i_{c}\right)
\end{aligned}
$$

with $\Delta=\left(1+c_{1} c_{2}\right) \int e d \tau$ is a constant.

2) The squared-voltage $y=x_{2}^{2}$ varies, in response to the tuning ratio $\beta$, according to the following equation: 


$$
\begin{aligned}
\dot{y}= & -\frac{L_{f}}{C_{f}} \hat{V}^{2}\left(1-\cos \left(2 \omega_{s} t\right)\right) \beta^{2} \\
& +\frac{1}{C_{f}}\left[\hat{V}^{2}\left(1-\cos \left(2 \omega_{s} t\right)\right)+2 L_{f} v_{s} i_{c}+L_{f} \Delta \hat{V} \sin \omega_{s} t\right] \beta \\
& -\frac{2 L_{f}}{C_{f}}\left(i_{c}^{2}+\frac{1}{L_{f}} v_{s} i_{c}+\Delta i_{c}\right)
\end{aligned}
$$

3) When $L_{f}$ can be neglected, the above relation becomes:

$$
\dot{y}=k_{o}\left(1-\cos \left(2 \omega_{s} t\right)\right) \beta-\frac{2}{C_{f}}\left(v_{s} i_{c}\right)
$$

with $k_{o}=\frac{\hat{V}^{2}}{C_{f}}$.

Proof. 1) Equation (29) is immediately obtained replacing, in (the second equation of) (5), $u$ by $u_{m}$ (given by (25)) and letting there $e=0$.

2) Equation (30) is easily obtained by deriving $y$ with respect to time and using (29).

3) Putting $L_{f}=0$ in equation (30) yields (31)

The ratio $\beta$ stands as a control input in the first-order system (31). The problem at hand is to design for $\beta$ a tuning law so that the squared voltage $y=x_{2}^{2}$ tracks a given reference signal $y^{*}=\left(x_{2}^{*}\right)^{2}$. Bearing in mind the fact that $\beta$ and its derivative should be available (Proposition 1), a PI control law is considered:

$\beta=c_{3} e_{3}+c_{4} e_{4}$

with $e_{3}=y^{*}-y, \quad e_{4}=\int_{0}^{t} e_{3} d \tau$

At this point, the regulator parameters $\left(c_{3}, c_{4}\right)$ are any positive real constants. The next analysis will make it clear how these should be chosen for the control objectives to be achieved.

\subsection{Control system analysis}

In the following Theorem, it is shown that the control objectives are achieved (in the mean) with an accuracy that depends on the network frequency $\omega_{s}$.

Theorem 1 (main result). Consider the shunt active power filter shown by Fig.1, represented by its average model (5), together with the controller consisting of the inner-loop regulator (25) and the outer-loop regulator (32). Then, the closed-loop system has the following properties:

1) Let the reference signal $y^{*}$ be any positive signal. There exists a positive real $\varepsilon^{*}$ such that if $\varepsilon \leq \varepsilon^{*}$ then, the tracking errors $e_{1}, e_{2}, e_{3}$ and the ratio $\beta$ are harmonics signals, with frequency, that continuously depends on $\varepsilon=1 / \omega_{s}$, i.e. $e_{1}=e_{1}(t, \varepsilon), e_{2}=e_{2}(t, \varepsilon), e_{3}=e_{3}(t, \varepsilon), \beta=\beta(t, \varepsilon)$.

2) Furthermore, when $\varepsilon \rightarrow 0$, all errors vanishes and $\beta$ converges:

$\lim _{\varepsilon \rightarrow 0} e_{1}(t, \varepsilon)=0 ; \quad \lim _{\varepsilon \rightarrow 0} e_{2}(t, \varepsilon)=0 ; \quad \lim _{\varepsilon \rightarrow 0} e_{3}(t, \varepsilon)=0$

$\lim _{\varepsilon \rightarrow 0} \beta(t, \varepsilon)=\frac{\hat{I}_{c 1}}{\hat{V}} \cos \varphi_{1}$

Proof. 1) First, notice that equation (32) guarantees that $\beta$ and its first time-derivative are available.

Then, by Proposition 1, the couple of errors $\left(e_{1}, e_{2}\right)$ undergoes equation (26). This together with (31) and (32) show that the augmented state vector $E_{o}=\left(\begin{array}{llll}e_{1} & e_{2} & e_{3} & e_{4}\end{array}\right)^{T}$ undergoes the following state equation:

$\dot{E}_{o}(t)=A(t) E_{o}(t)+P_{o}\left(t, y^{*}, v_{s}, i_{c}\right)$

where:

$$
\begin{gathered}
A(t)=\left(\begin{array}{cc}
A_{1}(t) & 0_{2,2} \\
0_{2,2} & A_{2}(t)
\end{array}\right) \\
P_{o}\left(t, y^{*}, v_{s}, i_{c}\right)=\left(\begin{array}{c}
0 \\
0 \\
\dot{y}^{*}+\frac{2}{C_{f}} v_{s}(t) i_{c}(t) \\
0
\end{array}\right)
\end{gathered}
$$

with

$$
\begin{aligned}
& A_{1}(t)=\left(\begin{array}{cc}
0 & 1 \\
-\left(1+c_{1} c_{2}-\omega_{s}^{2} \sin \left(\omega_{s} t\right)\right) & -\left(c_{1}+c_{2}+\omega_{s} \cos \left(\omega_{s} t\right)\right)
\end{array}\right) \\
& A_{2}(t)=\left(\begin{array}{cc}
-c_{3} k_{o}\left(1-\cos \left(2 \omega_{s} t\right)\right) & -c_{3} k_{o}\left(1-\cos \left(2 \omega_{s} t\right)\right) \\
1 & 0
\end{array}\right)
\end{aligned}
$$

The stability of the above system will now be dealt with making use of system averaging theory. To this end, introduce the time-scale change $\tau=\omega_{s} t$.

It is readily seen from (33) that $Z_{0}(\tau) \stackrel{\text { def }}{=} E_{0}(t)=E_{0}\left(\tau / \omega_{s}\right)$ undergoes the differential equation:

$$
\frac{d Z_{o}(\tau)}{d \tau}=\varepsilon A_{\tau}(\tau) Z_{o}(\tau)+\varepsilon P_{o \tau}\left(\tau, y^{*}, v_{s}, i_{c}\right)
$$

with:

$$
A_{\tau}(\tau)=\left(\begin{array}{cc}
A_{1 \tau}(\tau) & 0_{2,2} \\
0_{2,2} & A_{2 \tau}(\tau)
\end{array}\right)
$$




$$
\begin{aligned}
& A_{1 \tau}(\tau)=\left(\begin{array}{cc}
0 & 1 \\
-\left(1+c_{1} c_{2}-\frac{\sin (\tau)}{\varepsilon^{2}}\right) & -\left(c_{1}+c_{2}+\frac{\cos (\tau)}{\varepsilon}\right)
\end{array}\right) \\
& A_{2 \tau}(\tau)=\left(\begin{array}{cc}
-c_{3} k_{o}(1-\cos (2 \tau)) & -c_{3} k_{o}(1-\cos (2 \tau)) \\
1 & 0
\end{array}\right)
\end{aligned}
$$

Clearly, the above time-varying matrices are periodic with period $2 \pi$. Now, let us introduce the average functions:

$$
\begin{aligned}
& \bar{A}_{\tau}=\lim _{\varepsilon \rightarrow 0} \frac{1}{2 \pi} \int_{0}^{2 \pi} A_{\tau}(\tau) d \tau \\
& \bar{Z}_{o}=\lim _{\varepsilon \rightarrow 0} \frac{1}{2 \pi} \int_{0}^{2 \pi} Z_{o}(\tau) d \tau \\
& \bar{P}_{o \tau}=\lim _{\varepsilon \rightarrow 0} \frac{1}{2 \pi} \int_{0}^{2 \pi} P_{o \tau}\left(\tau, y^{*}, v_{s}, i_{c}\right) d \tau
\end{aligned}
$$

It follows from (35a-c) that:

$$
\begin{aligned}
& \bar{A}_{\tau}=\left(\begin{array}{cc}
\bar{A}_{1 \tau} & 0 \\
0 & \bar{A}_{2 \tau}
\end{array}\right) \\
& \bar{A}_{1 \tau}=\left(\begin{array}{cc}
0 & 1 \\
-\left(1+c_{1} c_{2}\right) & -\left(c_{1}+c_{2}\right)
\end{array}\right) \\
& \bar{A}_{2 \tau}=\left(\begin{array}{cc}
-c_{3} k_{o} & -c_{4} k_{o} \\
1 & 0
\end{array}\right) \\
& \bar{P}_{o \tau}=\left(\begin{array}{c}
0 \\
0 \\
\hat{V}_{c 1} \\
C_{f} \\
0
\end{array}\right)^{T} \varphi_{1}^{T}
\end{aligned}
$$

The last equality is obtained making use of the constant or periodic nature of the reference signal $y^{*}$. In order to get stability results regarding the system of interest (34), it is sufficient (thanks to averaging theory) to analyze the following averaged system:

$$
\dot{\bar{Z}}_{o}=\varepsilon \bar{A}_{\tau}+\varepsilon \bar{P}_{o \tau}
$$

To this end, notice that (37) has a unique equilibrium at:

$$
Z_{o}^{*}=\left(\begin{array}{c}
0 \\
0 \\
0 \\
\frac{\hat{V} \hat{I}_{c 1}}{c_{4}} \cos \left(\varphi_{1}\right)
\end{array}\right)
$$

On the other hand, as (37) is linear, the stability properties of its equilibrium are fully determined by the state-matrix $\bar{A}_{\tau}$. More specifically, the equilibrium $Z_{o}^{*}$ will be globally exponentially stable if the matrix $\bar{A}_{\tau}$ is Hurwitz. It is readily checked that $\bar{A}_{1 \tau}$ is Hurwitz whatever $c_{1}>0$ and $c_{2}>0$. Indeed, its characteristic polynomial is $\operatorname{det}\left(s I-\bar{A}_{1 \tau}\right)=s^{2}+\left(c_{1}+c_{2}\right) s+1+c_{1} c_{2}$ and a second order polynomial is Hurwitz if its coefficients are nonzero and of the same sign.

Similarly, $\bar{A}_{2 \tau}$ is Hurwitz whatever $c_{3}>0$ and $c_{4}>0$ because its polynomial characteristic, namely $\operatorname{det}\left(s I-\bar{A}_{2 \tau}\right)=s^{2}+c_{3} k_{0} s+c_{4} k_{0}$, has positive coefficients. The fact that $\bar{A}_{1 \tau}$ and $\bar{A}_{2 \tau}$ are both Hurwitz implies that the bloc diagonal matrix $\bar{A}_{\tau}$, given by (36a) is in turn Hurwitz. Then, the equilibrium $Z_{o}^{*}$ of the linear system (37) is actually globally exponentially stable. Applying e.g. Theorem 4.10 in (Khalil, 2003), one concludes that there exists a $\varepsilon^{*}>0$ such that for $\varepsilon<\varepsilon^{*}$, the differential equation (34) has a harmonic solution $Z_{0}=Z_{0}(t, \varepsilon)$ that continuously depends on $\varepsilon$. Moreover, one has $\lim _{\varepsilon \rightarrow 0} Z_{o}(t, \varepsilon)=Z^{*}$. This, together with (38), yields in particular that $\lim _{\varepsilon \rightarrow 0} e_{2}(t, \varepsilon)=0, \lim _{\varepsilon \rightarrow 0} e_{2}(t, \varepsilon)=0$, $\lim _{\varepsilon \rightarrow 0} e_{3}(t, \varepsilon)=0 \quad$ and $\quad \lim _{\varepsilon \rightarrow 0} e_{4}(t, \varepsilon)=\frac{\hat{V} \hat{I}_{c 1}}{c_{4}} \cos \left(\varphi_{1}\right) . \quad$ Then, using (32) one gets $\lim _{\varepsilon \rightarrow 0} \beta(t, \varepsilon)=\frac{\hat{I}_{c 1}}{\hat{V}} \cos \varphi_{1}$.

The Theorem is thus established.

\section{NUMERICAL SIMULATIONS}

The performances of the proposed controller are now numerically evaluated using a shunt APF based system with the following characteristics:

\begin{tabular}{l|l|l}
\hline \hline \multicolumn{2}{l|}{ PARAMETERS } & VALUES \\
\hline network & $v s$ & $220 \mathrm{~V}, 50 \mathrm{~Hz}$ \\
\hline Power active filter & $L_{f}$ & $3 \mathrm{mH}$ \\
& $C_{f}$ & $1000 \mu \mathrm{F}$ \\
\hline Rectifier & $L$ & $3 \mathrm{mH}$ \\
& $R$ & $2-5 \Omega$ \\
& $L_{o}$ & $100 \mathrm{mH}$ \\
\hline Current regulator & $c_{1}$ & 5000 \\
& $c_{2}$ & 5000 \\
\hline Voltage regulator & $c_{3}$ & $6.75 \times 10-7$ \\
& $c_{4}$ & $2.20 \times 10-5$
\end{tabular}

The simulation aim at illustrating the behavior of the controller in response to step changes on both the voltage reference $x_{2}^{*}$ and the nonlinear load resistance $R$. More specifically, the voltage reference goes from $600 \mathrm{~V}$ to $1000 \mathrm{~V}$.

The resulting controller performances are illustrated by Fig. 2 to 11 . As expected by Theorem 1, the output voltage $v_{d c}$ converges, in the mean, to its reference value with a good accuracy (Fig. 2). Furthermore, it is observed that the voltage ripples oscillates at the frequency $2 \omega_{s}$, but their amplitude are too small compared to the average value of the signals, confirming thus Theorem 1. Fig 3 shows that the network current $i_{s}$ is sinusoidal. Fig. 4 shows the waveform of the load current $i_{c}$; this clearly presents a harmonic distortion 
and phase shift with respect to $v_{s}$. In order to better appreciate the controller performances, a zoom is made in Figs 4 and 5 on the input current and network current.

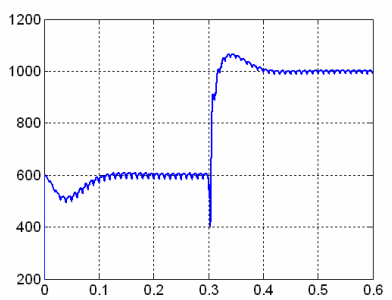

Fig.2 output voltage $v_{d c}$

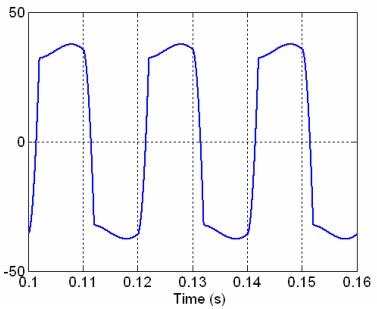

Fig.4 Load current $i_{c}$

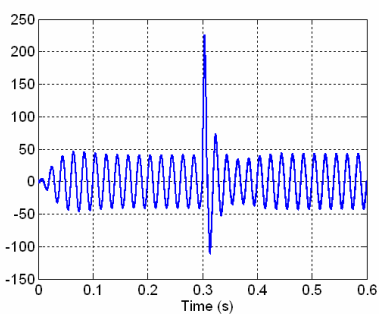

Fig.3 network current

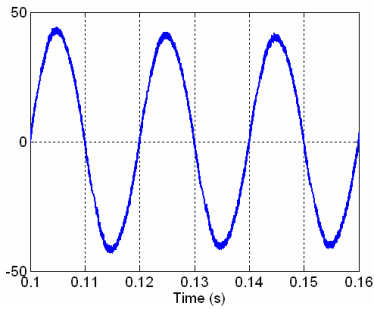

Fig.5 network current $i_{s}$

Figures 6 to 9 illustrate the behavior of the control system facing load changes that are not accounted for in the controller design. The rest of the converter characteristics are kept unchanged. The different figures show that the degradation in the controller performances remains very limited despite a $60 \%$ uncertainty on the load resistance. In particular, Fig. 8 shows that the output voltage regulation is recovered after a transient period following the load change. The steady-state tracking error is nearly 5\% (much smaller than load uncertainty). In turn, the control signal $\beta$ becomes rapidly constant (in the mean) after the load change.

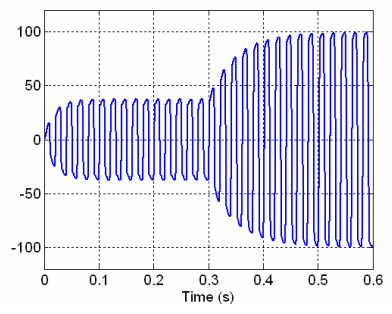

Fig. 6: load current $i_{c}$

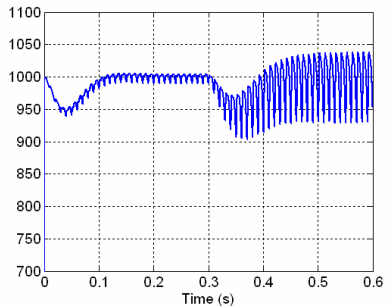

Fig.8: output voltage $v_{d c}$

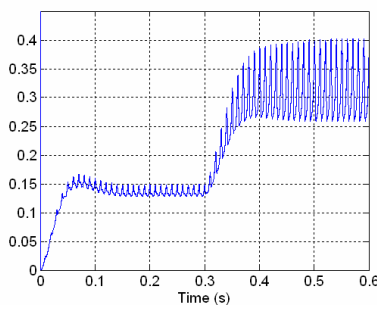

Fig. 7: control signal $\beta$

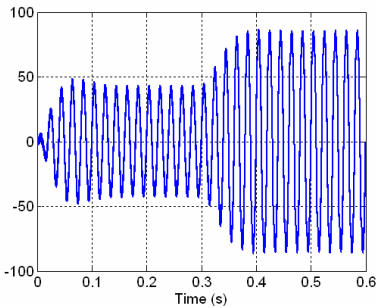

Fig.9: network current $i_{s}$

\section{CONCLUSION}

A nonlinear control strategy is proposed for single-phase shunt active power filters. A nonlinear two-loop cascade controller is developed to achieve current harmonics and reactive power compensation in presence of nonlinear and uncertain loads. Unlike former works, the controller is presently designed and analysed making use of advanced tools from the control theory e.g. backstepping design technique, system averaging theory. The theoretically proved performances of the controller are confirmed by several simulations that also show its robustness against load change and uncertainty.

\section{REFERENCES}

Gyugyi L., E. C. Strycula, (1976). Active AC Power Filters. IEEE/IAS Annu. Meeting, pp. 81-87.

Akagi H., (2005). Active Harmonic Filters, Proceedings of the IEEE, vol. 93, pp. 2128-2141.

Doğan H., Akkaya R., (2009), A Simple Control Scheme for Single-Phase Shunt Active Power Filter with Fuzzy Logic Based DC Bus Voltage Controller. IMECS, Vol II, Hong Kong.

Hassan K. Khalil, (2003). Nonlinear systems. Prentice Hall, 3 th edition.

Costa-Castelló R., Griñó R., Cardoner R., Fossas E. (2007), High Performance Control of a Single-Phase Shunt Active Filter. IEEE International Symposium on Industrial Electronics, Vigo, Spain, pp. 3350 - 3355.

Krein P.T., J. Bentsman, R.M. Bass and B. Lesieutre (1990). On the use of averaging for analysis of power electronic system. IEEE Trans. Power Electronics, Vol. 5, $\mathrm{n}^{\circ}$ 2, pp. 182-190.

Andrieu, C., J. P. Ferrieux and M. Rocher (1996). The ac/dc stage: A survey of structures and chopper control modes for power factor correction. EPE J., vol. 5, pp. 17-22.

Tse, C. K. and M. H. L. Chow (2000). Theoretical study of switching converters with power factor correction and output regulation. IEEE Trans. Circuits Syst. I, vol. 47, pp. 1047-55.

Erickson, R., M. Madigan and S. Singer (1990) Design of simple high power factor rectifier based on the flyback converter. IEEE Applied Power Electronics Conference and Exposition, Los Angeles, CA, USA, pp. $792-801$.

Karagiannis, D. E. Mendes, A. Astolfi, and R. Ortega (2003). An experimental comparison of several PWM controllers for a single-phase $A C-D C$ converter. IEEE Trans. Contr. Syst. Technol., vol. 11, pp. 940-947.

Abouloifa, A., F. Giri, I. Lachkar and F. Z Chaoui (2010). Formal Framework for Nonlinear Control of PWM AC/DC Boost Rectifiers - Controller Design and Average Performance Analysis. IEEE Trans. Contr. Syst. Technol., Vol. $18 \mathrm{~N}^{\circ}$ 2, pp. 323 - 335

J. Miret, L. Garcia de Vicuña, J. Matas, J. M. Guerrero, J. Cruz, (2004), Simplified Feedback Linearization of a Single-Phase Active Power Filter Using Sliding Mode Control, International Symposium on Industrial Electronics, Maig. 